\title{
A Distributed Control System using CAN bus for an AUV
}

\author{
Wenbao Geng ${ }^{a}$, Yu Huang ${ }^{b}$, Peng Lu ${ }^{c}$ \\ No. 710 R\&D Institute, CSIC, Yichang 443003 China \\ a15271531882@163.com, ${ }^{\mathrm{b}}$ mechanicalgeng@163.com, '9upeng@163.com
}

Keywords: CAN bus, distributed control system, AUV, ladder architecture, autonomous navigation.

\begin{abstract}
A new distributed control system using CAN bus for an AUV is proposed in this paper. According to its characteristic, the control system is divided into functional independent control module and various modules acted as CAN bus node form the whole communication network. The console assigns a mission and then central control unit centralizes management and decentralizes control using ladder architecture. The paper makes a detailed introduction of AUV's Autonomous navigation experiment using this control system. In the real experiment, AUV executed task assigned by console quickly and accurately, fully showing the applicability of this control system.
\end{abstract}

\section{Introduction}

Autonomous Underwater Vehicle (AUV) is a kind of underwater robot, which is intelligent, and realize autonomous navigation and various functions [1].Due to the insecurity and uncertainty of underwater work, AUV has become the main tool for detecting underwater field. For example, Hydroid Lockheed's REMUS series UUV, Bluefin robotics' Bluefin series UUV [2], etc , has played an important role in the field of underwater detection.

We developed an underwater unmanned platform, mainly used for carrying acoustic equipment, simulating acoustic source, measuring underwater environment, etc. The specific structure is shown in Fig. 1. The AUV navigation system uses integrated navigation method of strapdown inertial navigation, DVL (Doppler velocity $\log$ ) and GPS to ensure the precision of navigation. Control system and propulsion system respectively uses servo control and motor control. Communication system uses a combination of radio and Beidou, by using the effective ones to ensure the reliability of communication. Emergency system uses ballast release way to ensure the safety of equipment, device controller feedbacking status information and the AD collecting signals to determine whether the need of emergency. Descent uses the manner of horizontal rudder control and slide forward auxiliary dive to ensure AUV dive rapidly. The AUV requires high navigation accuracy, high autonomy, and state storage, information interaction, emergency protection and some other column functions so that the AUV control task is very complicated. In this case, single controller and the traditional centralized control mode will make the controller tasks too heavy, and have low efficiency [3].

A new distributed control system using CAN bus for an AUV is proposed and designed in this paper; separate controllers are designed for each module completing the information processing and the underlying local control to ensure the reliability of communication and flexibility [4]. And each module uses ladder architecture, the console assigning a mission and then central control unit centralizing management, to ensure the effectiveness and orderliness of the control system. In the real experiment, AUV completed autonomous navigation task safely and reliably which showing the applicability of this control system. 


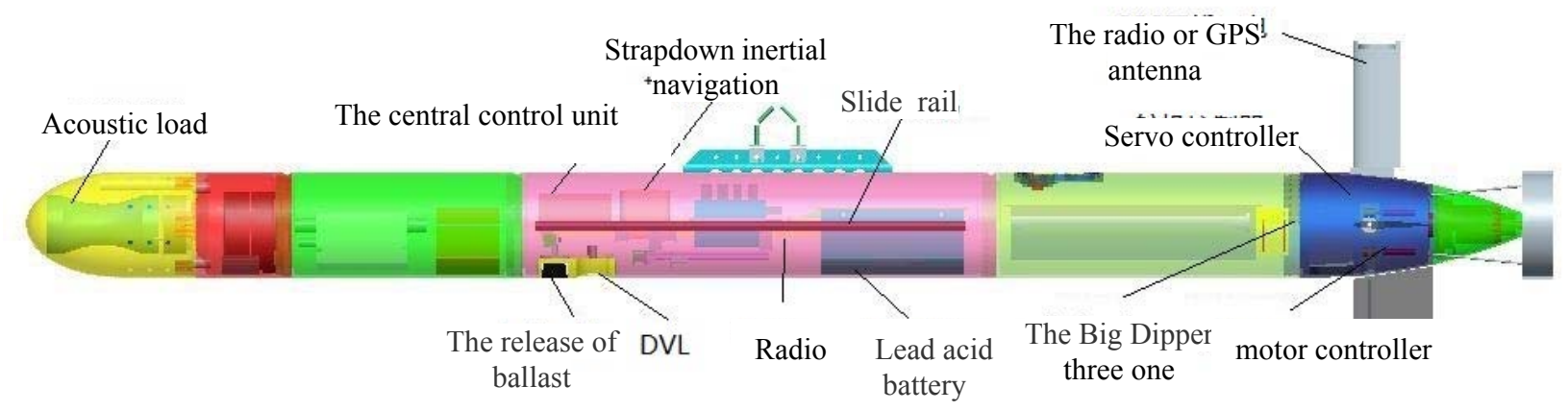

Fig. 1 General schematic of the unmanned carrying platform

\section{CAN bus control system based on AUV model}

CAN bus is a serial communication network which is the most used and most effective in the real time control system or distributed control system. Compared with other distributed system, CAN bus works in the multi master mode, each node in the network can be sent to the bus data according to the priority and high communication speed can reach with $1 \mathrm{M} / \mathrm{S}$ that is very real-time. The biggest feature is the use of communications data block coding method, the number of communication nodes on the bus is not restricted, actually the number of node can constitute up to 110 [5].

Underwater vehicle carrying platform control system is a distributed control system based on CAN bus and mainly includes communication system, power and control system, navigation system, emergency system, signal acquisition system and load system, etc. The architecture of distributed control system is shown in Fig. 2. Among them, the water control system is mainly the ground control equipment of AUV and realizes the information interaction with the central control unit through radio or Beidou communication module. The remaining system is located inside the aircraft and exchanges information with the central control unit mainly through the controller and sensor respective. During AUV runtime, surface console sends task instructions to the central control unit, the central control unit will decompose sail task into the control instruction that bottom controller can understand and will be transmitted to the underlying controller by bus, and then the bottom controller drives the actuator's movement, this process forms the distributed structure of ladder. In addition, the central control unit receives data feedbacking from the bottom controller, and memorizer records and stores data constantly.

At present, the highest accuracy of AUV navigation is the mode of strapdown inertial navigation, DVL (Doppler velocity log) and GPS integrated navigation [6], when underwater operating, due to GPS information can not be received, underwater vehicle carrying platform adopts the mode of inertial navigation and DVL navigation. DVL sends real-time speed information through the serial port to inertial navigation and inertial navigation dead reckons based on the data obtained , and transmits the inertial navigation information of latitude and longitude, the attitude information, status information to the central control unit through the CAN bus in real-time .

Emergency system is to guarantee the security of AUV. Each controller feedback real-time the respective status information through CAN bus, such as whether steering mechanism is normal, whether the motor overvoltage, etc. And each sensor is also continuously detecting depth information, the height from the bottom and water Leakage information of the aircraft. According to the different types of fault, the central control unit will make in real time emergency measures to control the action of steering engine, motor and the release of ballast.

The power and control system mainly comprises a motor controller, the steering engine controller and actuator. Actuating mechanism mainly comprises a steering mechanism, a motor and a slider. The central control unit issues autonomous navigation instructions, the steering controller and motor controller will receive the rudder angle instruction and speed commands into voltage signal to the various implementing agencies and feedback the real-time state information. When it needs to dive, 
the central control unit will send electrical instructions to control slider's slide action to realize the auxiliary dive.

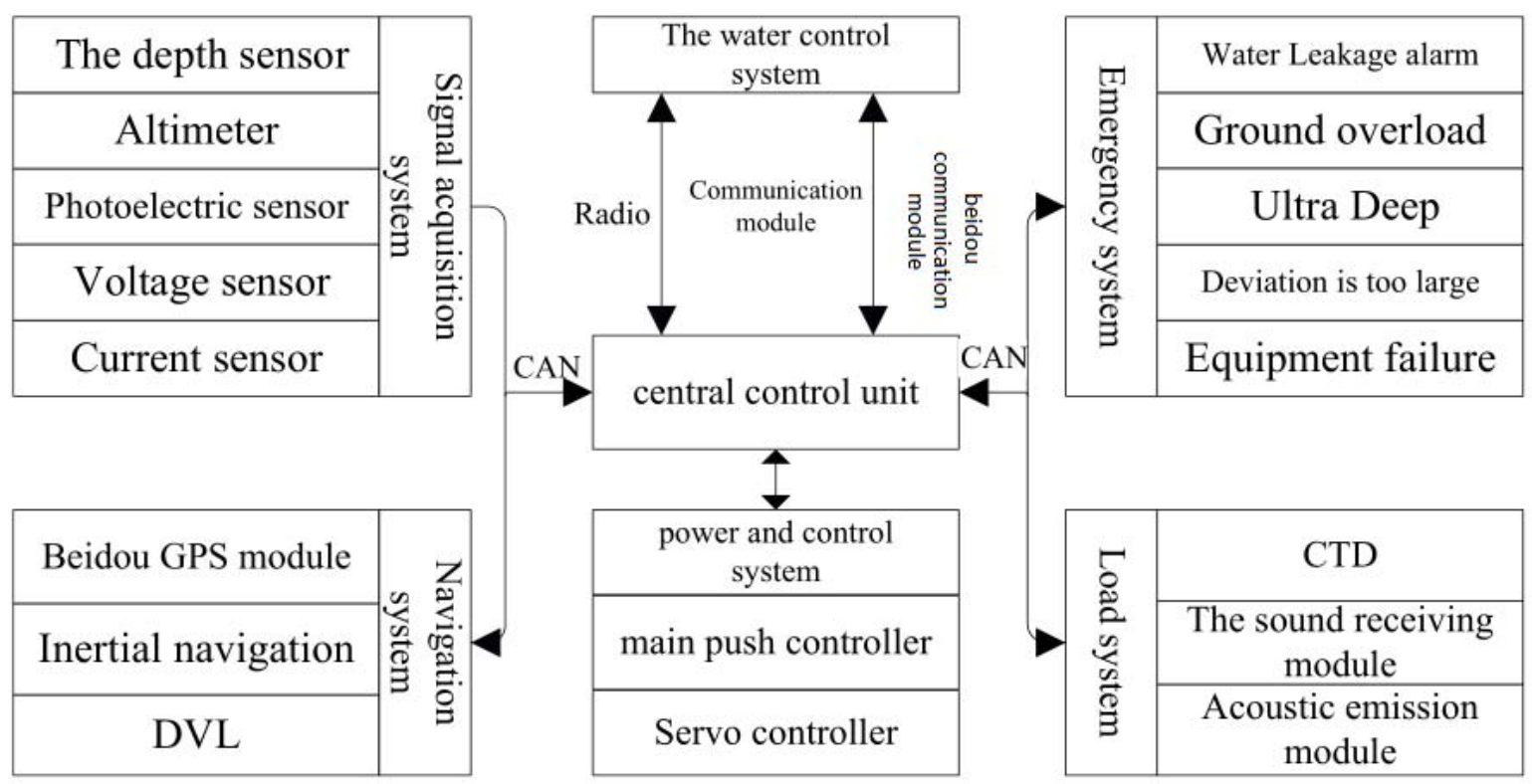

Fig. 2 Distributed control system

\section{AUV autonomous navigation control process}

The main task of AUV autonomous navigation is sailing according to the point GPS specified by console. The main work includes path planning, mode selection, course control, track control and depth control. The concrete work flow is shown in Fig. 3.

When performing a task, the water control system (abbreviated as PC) assigns task instruction to the central control unit (referred to as the client) by radio or Beidou communication module, the contents of the task includes the target position, the depth of the target and the time of sailing. When the instruction is issued, the lower position machine will feedback task success marks to the console. After the instruction is issued, the lower position machine feedback task success marks to the console. Then the host computer startup s autonomous mode and AUV begin to take autonomous navigation. First, the power and control system control AUV dive, the slider will move forward and the centre of gravity of AUV will move forward to assist diving. After the AUV completely diving, depth gauge and navigation control center will real-time update current depth and location, AUV execute the motion control, on the longitudinal direction it uses the double closed-loop control structure of the pitch angle-the depth [7] to make AUV reach a predetermined depth. And on the horizontal direction it uses the combination of Yaw distance and heading angle control structure to make AUV reach a predetermined position. When it reaches the target point, AUV will start to sail down to the following target point until it reaches the final target point, then AUV floats up and will upload data stored in memory to the console. During navigation process, the lower position machine detects the running state of AUV in real time, when it comes to overtime or yaw angle is too large (more than 200m) and other failures, emergency system will terminate the current operation and AUV will come-up peremptorily. 


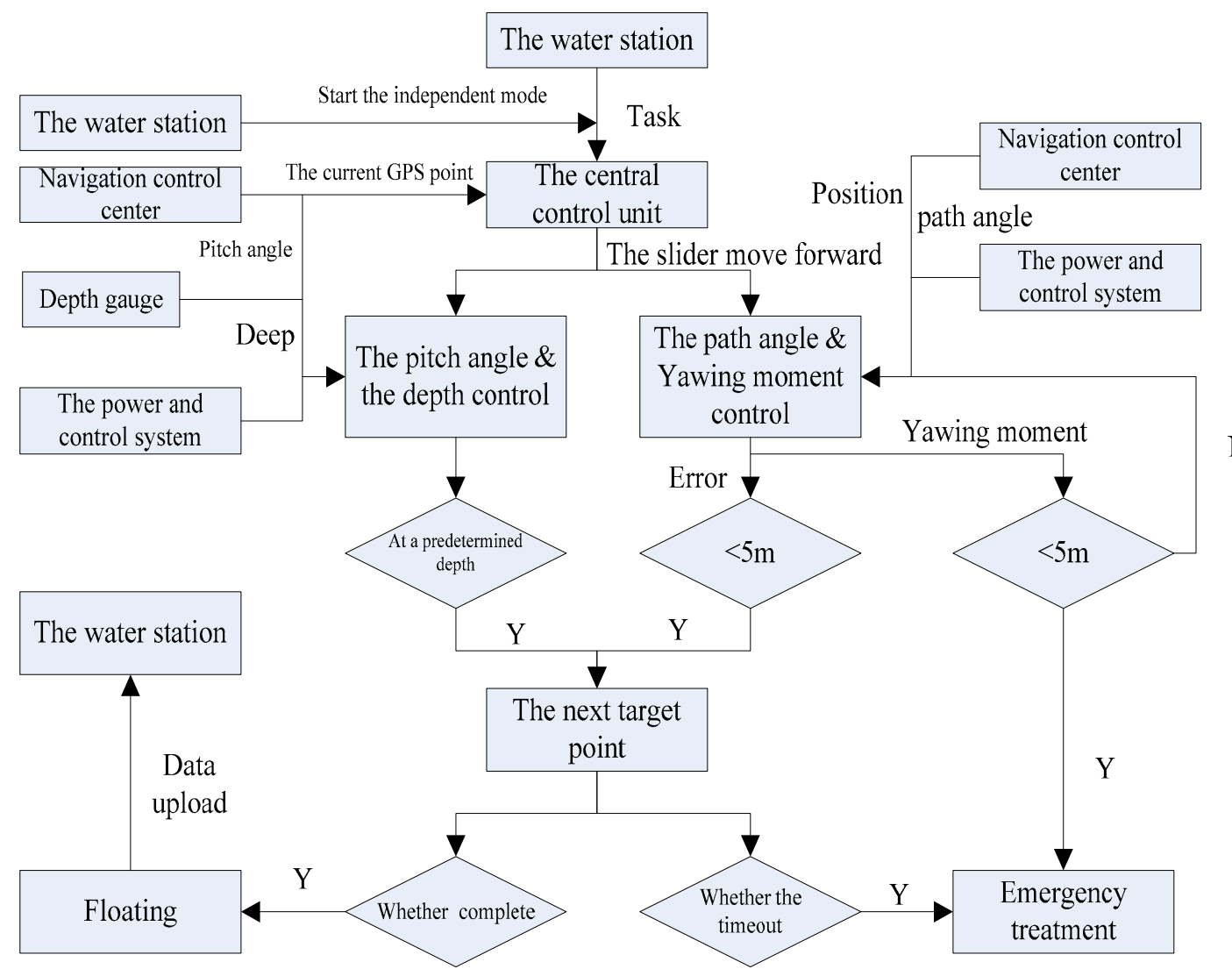

Fig. 3 Autonomous navigation tree of AUV

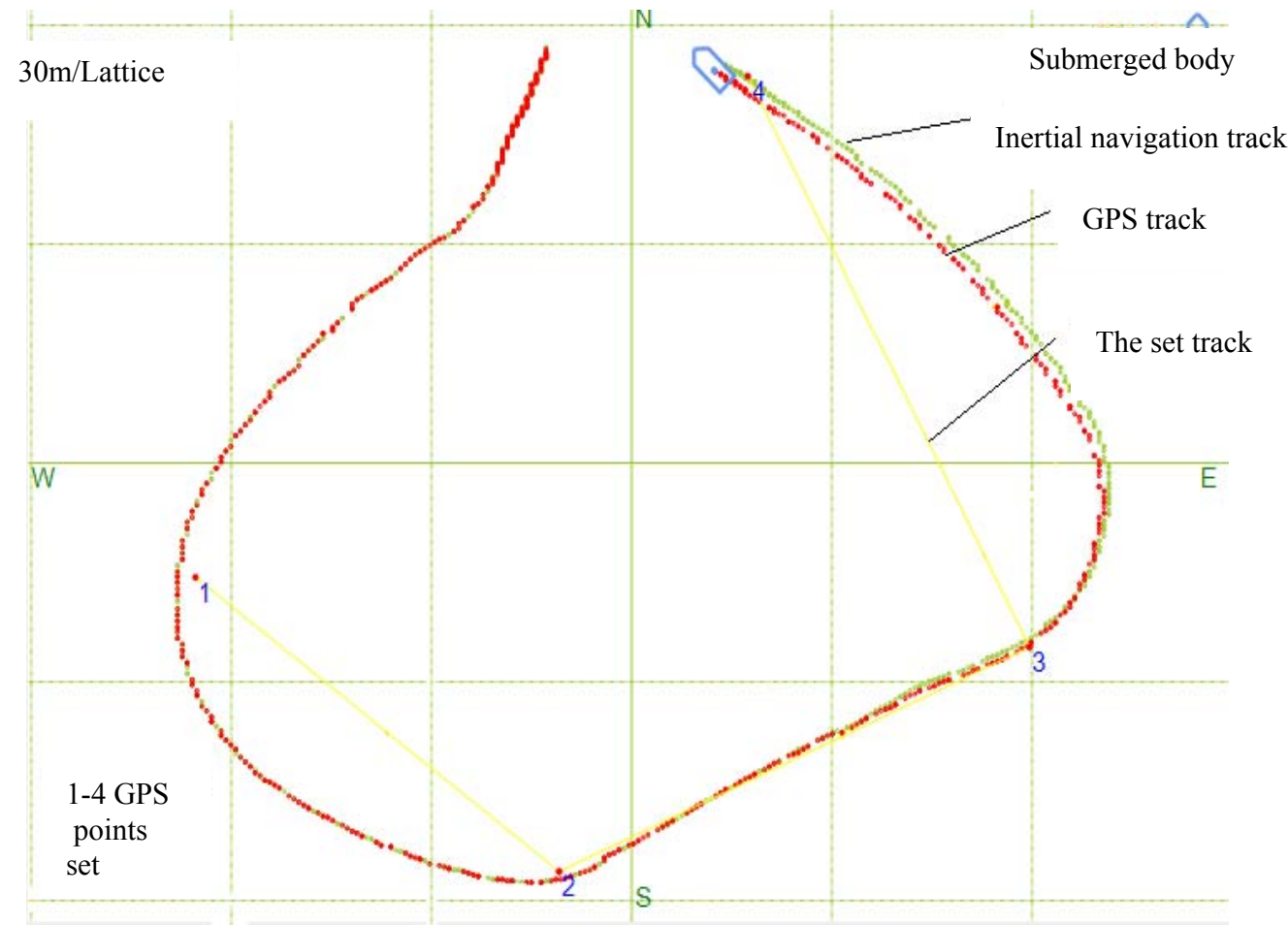

Fig. 4 The experiment results of autonomous navigation

\section{Experiment results}

Running point accuracy of this experiment is set to $5 \mathrm{~m}$. Below the 2.5 section speed, AUV's turning radius is $15 \mathrm{~m}$, the distance of GPS's two points is set to approximately $100 \mathrm{~m}$. In order to testing the track and the precision of navigation of AUV underwater running, operating depth is set to $0 \mathrm{~m}$, the navigation mode uses DVL integrated navigation (inertial navigation uses the information of DVL 
speed to estimate position of AUV). The results of autonomous navigation experiment is shown in Fig. 4. When there is a slight deviation between the running track of inertial navigation and GPS's track, yawing moment will become smaller and smaller and the AUV will be able to reach the specified target GPS point. (1, 2, 3, 4 points on a graph). In the experiment process, AUV operated normally and reliably, it completed assigned task well which fully shows the applicability of this kind of distributed control system.

\section{Conclusion}

A new distributed control system using CAN bus for an AUV is proposed in this paper and has been used to underwater unmanned platform. Each module of underwater unmanned platform has its own controller and forms a network through the CAN bus. Each module is independent of each other without mutual interference, designing based on the CAN bus makes the control system have good maintainability and scalability. The central control unit no longer directly controls actuator but as a unified coordination and dispatching center, and it will decompose the instruction into the control instruction that bottom controller can understand, then the underlying controller controls the actuator's movement. The distributed control system reduces the work load of the central control unit and improves the working efficiency of the central control unit. In the experiment of autonomous navigation, AUV completed tasks fast and accurately fully demonstrating the practicability of this control system.

\section{References}

[1] Xu Yuru, Li Pengchao, Development trend of underwater robot[J]. Journal of natural, 2011, 33(3):126-127.

[2] Zhao Tao, Liu Mingyong, Zhou Liangrong. Research status and challenges of autonomous underwater vehicle [J]. Fire control and command,2010,35(6):1-2.

[3] Wang Gang. AUV control system based on Multi sensor[D]. Harbin Engineering University, 2013

[4] Gao jian, Yan Weisheng, Zhang Fubin. A distributed control for autonomous navigation underwater[J]. Journal of Materials Engineering,2009,30(8):1140-1141

[5] Wu Kuanming. The principle of CAN bus and design of application system[M]. Beihang University press. 1996

[6] Yan Weisheng, Xu Deming, Li Jun. Underwater autonomous navigation technology [J]. Fire control and command,2004,29(6):14-15.

[7] $\mathrm{Xu}$ Demin. Automatic control system of the torpedo [M]. Northwestern Polytechnical University press. 2000 